\title{
Predicción de estrés térmico en vacas lecheras mediante indicadores ambientales y fisiológicos
}

\author{
Cerqueira, J.O.L. ${ }^{1,5}$; Araújo, J.P.P. ${ }^{1,2}$; Blanco-Penedo, I. ${ }^{3}$; Cantalapiedra, J. ${ }^{4}$; Silvestre, A.M.D. ${ }^{5}$ y Silva, S.J.C.R. ${ }^{5}$
}

\author{
'Escola Superior Agrária do Instituto Politécnico de Viana do Castelo. Ponte de Lima. Portugal. \\ ${ }^{2}$ Centro de Investigação de Montanha (CIMO) - ESA-IPVC. Portugal. \\ ${ }^{3}$ Subprograma Bienestar Animal. IRTA. Monells. Girona. España. \\ ${ }^{4}$ Servicio de Ganadería de Lugo. Xunta de Galicia. España. \\ ${ }^{5}$ CECAV - Universidade de Trás-os-Montes e Alto Douro. Vila Real. Portugal.
}

\section{Palabras ClaVe ADICIONALES}

\section{Holstein-Frisia.}

Índice de temperatura humedad.

Frecuencia respiratoria.

Temperatura rectal.

Producción de leche.

\section{RESUMEN}

Las vacas lecheras son animales muy sensibles a los cambios ambientales y requieren alojamientos cada vez más sofisticados para prevenir la aparición de estrés térmico en épocas calurosas que eviten importantes pérdidas económicas por la disminución de producción. El objetivo del presente estudio consistió en la valoración en explotación de indicadores ambientales y de indicadores fisiológicos de estrés de las vacas y su influencia sobre la producción de leche. Se monitorizaron la temperatura y la humedad relativa del aire a intervalos de una hora, a través de sondas digitales colocadas en 4 explotaciones durante 365 días. Paralelamente se recogieron indicadores fisiológicos (temperatura rectal y frecuencia respiratoria) en los animales, en invierno y en verano. Los resultados mostraron temperaturas superiores a $25^{\circ} \mathrm{C}$ durante 48 días del año, siendo los períodos más críticos primavera y verano. La humedad relativa tuvo una variación de 10\% entre invierno y verano. El índice temperatura humedad (ITH) superó el valor de 72 en 51 días anualmente y el período más crítico de la jornada fue entre las 12:00 y las 16:00 horas (P4), con un ITH medio de 68,0. El paso del ITH de 72 por encima de 78 significó un incremento de $1,3^{\circ} \mathrm{C}$ y 37,3 respiraciones por minuto en las vacas lecheras. Se encontró una correlación alta entre la frecuencia respiratoria, temperatura rectal y ambas con el ITH, por lo que estos indicadores fisiológicos resultan ser excelentes predictores de estrés térmico. Los animales expuestos a ITH superior de 78 sufrieron una pérdida de producción de leche de 1,8 kg/vaca/día.

\section{Prediction of heat stress in dairy cows by environmental and physiological indicators}

\section{ADDITIONAL KEYWORDS}

Holstein-Friesian.

Temperature humidity index.

Respiratory rate.

Rectal temperature.

Milk production.

INFORMACIÓN

Cronología del artículo.

Recibido/Received: 28.09.2015

Aceptado/Accepted: 22.06.2016

On-line: 15.09 .2016

Correspondencia a los autores/Contact e-mail:

cerqueira@esa.ipvc.pt

\section{SUMMARY}

Dairy cows have become very sensitive animals to environmental changes, requiring increasingly sophisticated accommodations to prevent the stress that would lead to major production and economic losses. The aim of this study was to assess environmental and physiological indicators of stress in cows and their influence on milk production. The temperature and relative humidity was monitored at intervals of one hour through digital probes placed in four farms for 365 days. Alongside two animal physiological indicators (rectal temperature and respiratory rate) were collected, in winter and summer. The results showed the presence of 48 days in a year with temperatures above $25^{\circ} \mathrm{C}$, with the most critical periods occurring in spring and summer. The relative humidity ranged from just $10 \%$ between winter and summer. The Temperature Humidity Index (THI) exceeded the value of 72 on 51 days per year. The most critical period of the day recorded was between 12:00 to 16:00 p.m. (P4) where the average of THI was 68.0. From THI value below 72 to above 78 , a rise of $1.3^{\circ} \mathrm{C}$ and 37.3 breaths/minute was observed in the monitored dairy cows. We found a high correlation between respiratory rate and rectal temperature and of both with the $\mathrm{THI}$, highlighting these physiological indicators as excellent predictors of heat stress. The animals exposed to higher THI than 78 showed a loss in milk production of $1.8 \mathrm{~kg} / \mathrm{cow} /$ day.

\section{INTRODUCCIÓN}

Las condiciones ambientales, principalmente la elevada temperatura y humedad relativa en vacas lecheras provocan un impacto negativo sobre su producción, comportamiento, salud y bienestar. Un ambiente demasiado caliente puede afectar negativamente a la producción de leche en vacas lecheras, especialmente en animales de alto valor genético (Kadzere et al., 2002) y tiene efectos negativos sobre su bienestar (Jacobsen, 1996). La temperatura ambiente es probablemente el factor más investigado y al mismo tiempo el más ampliamente utilizado como un indicador de estrés en las vacas lecheras. 
Las condiciones climáticas ideales para la producción de leche se producen a temperatura ambiente entre $5^{\circ}$ y $25^{\circ} \mathrm{C}$, siendo éste intervalo considerado la zona de confort térmico. A su vez la tolerancia de las vacas a temperaturas inferiores a $5^{\circ} \mathrm{C}$ depende de la edad y el nivel de producción láctea.

Una vaca adulta en el pico de lactación es más tolerante al frío en función del calor producido por la fermentación ruminal y el metabolismo de los nutrientes. Por otro lado, el rendimiento de las vacas disminuye rápidamente a medida que la temperatura supera los $27^{\circ} \mathrm{C}$, independientemente de su edad, etapa de lactación o la humedad relativa del aire (Kadzere et al., 2002).

Cuando la temperatura supera los $27^{\circ} \mathrm{C}$ el porcentaje de vacas tumbadas en el suelo disminuye, aumentando el número de vacas de pie principalmente en el corredor de alimentación en una postura que les facilita la disipación de calor (Overton et al., 2002). Estos resultados fueron corroborados por Zahner et al .(2004) al observar que la duración del comportamiento de vacas acostadas disminuía durante el día, en sentido inverso al aumento de ITH, aunque el comportamiento de descanso de los animales durante la noche no se veía afectado.

Sin embargo no es únicamente la temperatura del aire la responsable de la variación de la temperatura corporal de las vacas, ya que hay otros factores, como la humedad relativa, velocidad del aire y la temperatura de la superficie de la cama, paredes y suelo del establo (Ramos, 2009). Cuando la temperatura ambiente en el establo es adecuada, el rango de humedad debe estar comprendido entre el 40 y $70 \%$ y preferentemente entre 50 y $60 \%$.

Cuando la humedad es alta, hay una disminución en el potencial de disipación de calor, tanto por la piel como por las vías respiratorias, que afectan a los animales con destaque en los ambientes en los cuales la eliminación de calor por evaporación es esencial para mantener la condición homeotérmica (Reece, 2009).

El ITH combina la temperatura y la humedad relativa en un índice único, que es comúnmente asociado con el estrés térmico. El ITH es ampliamente utilizado en las regiones cálidas de todo el mundo, para evaluar el impacto del estrés calórico en vacas lecheras (Bouraoui, 2002).

Según Johnson (1985) y DuPreez et al. (1990), la producción de leche no se ve afectada por el estrés térmico cuando los valores de ITH están entre 35 y 72. Sin embargo la producción de leche y el consumo de alimentos concentrados empiezan a disminuir cuando la ITH alcanza el valor de 72 (Armstrong, 1994; Silva et al., 2002) y esos dos parámetros disminuyen drásticamente cuando la ITH alcanza valores a partir de 76 (Johnson, 1980).

La elevación de la ITH tiene un efecto rápido sobre la reducción de la producción. Reiczigel (2009) y Solymosi et al. (2010) demostraron que un solo día en el que los animales son sometidos a estrés térmico, es probable que causen una caída en promedio de 1,5 a 2,0 kg de leche/vaca/día (5-10\% de la producción diaria).
Generalmente en respuesta a estrés por calor, se produce una reducción en el consumo de alimentos y producción de leche y un aumento de la temperatura corporal y frecuencia respiratoria de los animales (West, 2003; Correa-Calderón et al., 2009).

Para vacas lecheras de alta producción en equilibrio térmico, la temperatura rectal es independiente de la temperatura del aire, relacionándose con el metabolismo energético (Martello et al., 2009).

Los cambios en la temperatura rectal y la frecuencia respiratoria son los dos parámetros fisiológicos más comúnmente utilizados para medir el confort animal y la capacidad de adaptación a ambientes adversos (Hemsworth et al., 1995), mientras que el ITH se ha usado para evaluar el impacto ambiental de ganado porque puede describir más exactamente los efectos del medio ambiente sobre la capacidad de los animales para disipar el calor (West, 1999). Un aumento de la tasa de claudicación al final del verano se ha asociado a periodos de estrés por calor (Cook et al., 2004).

El objetivo de este trabajo consistió en la obtención de indicadores ambientales y fisiológicos capaces de identificar las vacas lecheras con estrés térmico y determinar las pérdidas de producción de leche.

\section{MATERIAL Y MÉTODOS}

\section{EXPLOTACIONES}

Para este trabajo fueron seleccionadas cuatro explotaciones del norte de Portugal (Ponte de Lima, Viana do Castelo y dos en Barcelos) con un sistema de producción intensivo libre con cubículos y con ventilación natural, que carecían de sistemas artificiales. Los censos de las explotaciones oscilaron entre 48 y 240 animales (tabla I).

\section{Medición de LA teMPERATURA Y HUMEDAD RELATIVA}

Se colocaron 2 sondas (Tinytag ultra 2-TGU-4500) por explotación para medir la temperatura y humedad relativa, fijándose a una altura aproximada de $2 \mathrm{~m}$ en dos áreas distintas del establo, en la zona de los cubículos y al lado del comedero. Las sondas registraron los parámetros ambientales a intervalos de una hora, durante un año.

\section{CÁlCulo del índice de temperatura-humedad (ITH)}

El ITH se calculó con la ecuación de Kibler (1964): $\mathrm{ITH}=1,8 \mathrm{Ta}-(1-\mathrm{HR})(\mathrm{Ta}-14,3)+32$, donde la Ta es la temperatura media $\left({ }^{\circ} \mathrm{C}\right)$ y $\mathrm{HR}$ es la humedad relativa

Tabla I. Explotaciones visitadas, tamaño del rebaño y número de animales observados (Farms visited, herd size and number of animals observed).

\begin{tabular}{cccc}
\hline Explotaciones & $\begin{array}{c}\text { Vacas en } \\
\text { producción }\end{array}$ & $\begin{array}{c}\text { Animales } \\
\text { observados }\end{array}$ & Repeticiones \\
\hline 1 & 240 & 13 & 4 \\
2 & 48 & 16 & 4 \\
3 & 52 & 13 & 4 \\
4 & 56 & 11 & 4 \\
4 & 396 & 53 & 16 \\
\hline
\end{tabular}


promedio, expresada como un porcentaje. Espinoza et al. (2011), afirman que cuando el ITH es superior a 72, las vacas lecheras de alta producción están muy afectadas y clasifican las variables de respuesta al ITH en tres categorías: $<72, \geq 72<78$ e $\geq 78$

\section{INDICADORES FISIOLÓGICOS DEL ESTRÉS}

Al mismo tiempo se recogieron indicadores de estrés térmico en 53 animales tomados al azar de entre los distintos niveles de producción, en cuatro ocasiones diferentes en cada explotación: dos en el verano y dos en el invierno durante el ordeño de la tarde (tabla I).

La temperatura rectal se obtuvo a través de un termómetro al comienzo del ordeño y al mismo tiempo se registró su frecuencia respiratoria. Durante la realización del estudio se registró la producción de leche de cada animal al final del ordeño y la producción diaria total de cada explotación.

\section{ANÁLISIS ESTADÍSTICO}

Para el análisis estadístico fue utilizado el programa SPSS para Windows versión 19 (SPSS Inc.). Se hizo el cálculo de estadísticas descriptivas, tanto de tendencia central (media), como la dispersión (desviación estándar y coeficiente de variación) en los diferentes indicadores, elaborándose gráficas e histogramas para una mejor comprensión de los resultados obtenidos.

Se aplicaron modelos de regresión lineal, análisis de varianza simples, dobles y triples, con interacciones y covariable en algunos análisis. Para la comparación múltiple de medias se aplicó la prueba de Tuckey.

\begin{tabular}{|c|c|c|c|c|}
\hline Parámetros & Primavera & Verano & Otoño & Invierno \\
\hline $\mathrm{N}$ & 17664 & 17664 & 17472 & 21106 \\
\hline $\begin{array}{l}\text { Temperatura } \\
\text { mínima }\left({ }^{\circ} \mathrm{C}\right)\end{array}$ & 7,4 & 11,2 & 3,7 & 0,6 \\
\hline $\begin{array}{l}\text { Temperatura } \\
\text { máxima }\left({ }^{\circ} \mathrm{C}\right)\end{array}$ & 34,4 & 37,1 & 33,4 & 26,5 \\
\hline $\begin{array}{l}\text { Temperatura } \\
\text { media }\left({ }^{\circ} \mathrm{C}\right)\end{array}$ & $19,5^{\mathrm{a}} \pm 5,2$ & $22,1^{\mathrm{b}} \pm 4,9$ & $15,8^{c} \pm 5,3$ & $11,6^{\mathrm{d}} \pm 4,2$ \\
\hline $\begin{array}{l}\text { Días de } \\
\text { temperatura } \\
>25^{\circ} \mathrm{C}\end{array}$ & 16,3 & 24,4 & 6,9 & 0,3 \\
\hline $\begin{array}{l}\text { Humedad } \\
\text { mínima (\%) }\end{array}$ & 21,4 & 26,6 & 27,0 & 26,0 \\
\hline $\begin{array}{l}\text { Humedad } \\
\text { máxima (\%) }\end{array}$ & 98,1 & 95,3 & 99,3 & 98,9 \\
\hline $\begin{array}{l}\text { Humedad } \\
\text { media (\%) }\end{array}$ & $71,3^{a} \pm 18,6$ & $70,1^{\mathrm{a}} \pm 14,9$ & $79,4^{b} \pm 15,0$ & $80,4^{b} \pm 16,3$ \\
\hline ITH & $64,7^{\mathrm{a}} \pm 6,7$ & $68,8^{b} \pm 6,0$ & $59,5^{c} \pm 7,6$ & $52,8^{d} \pm 6,7$ \\
\hline $\begin{array}{l}N^{\circ} \text { de días } \\
\text { ITH }>72\end{array}$ & 15,8 & 28,4 & 6,9 & 0,1 \\
\hline
\end{tabular}

ITH - índice de temperatura-humedad. En los componentes analizados valores de letra distinta $(a \neq b \neq c \neq d)$ son significativamente diferentes $(p<0,05)$.

\section{RESULTADOS}

Se encontraron diferencias significativas $(p<0,05)$ en la temperatura entre todas las estaciones del año. La temperatura más baja fue observada en invierno $(11,6$ $\left.\pm 4,2^{\circ} \mathrm{C}\right)$, estando este valor duplicado en el verano $\left(22,1 \pm 4,9^{\circ} \mathrm{C}\right)$, con temperaturas máximas de $37,0^{\circ} \mathrm{C}$. En primavera y en otoño a pesar de que la temperatura media fue de 19,5 y $15,8^{\circ} \mathrm{C}$ respectivamente, se encontraron valores máximos de aproximadamente $34,0^{\circ} \mathrm{C}$. Durante todo el año se contabilizaron 48 días donde la temperatura se elevó por encima de $25^{\circ} \mathrm{C}$, con mayor énfasis en las estaciones de verano y la primavera con 24 y 16 días respectivamente.

Por otro lado, la humedad relativa no mostró diferencias $(p>0,05)$ entre primavera y verano y entre otoño e invierno, variando unos 10 puntos porcentuales entre estos dos grupos de estaciones, con valores de 70,1 \pm $14,9 \%$ y $80,4 \pm 16,3 \%$ en verano e invierno respectivamente. Como era de esperar para el valor de ITH se encontraron diferencias $(\mathrm{p}<0,05)$ entre todas las estaciones, con el menor valor en invierno $(52,8 \pm 6,7)$ y el más elevado en verano $(68,8 \pm 6,0)$. Este indicador fue superior a 72 en 51 días del año, con 28 días en verano y 16 días en primavera (tabla II).

La humedad relativa osciló entre el $67 \%$ en junio y el $87 \%$ en diciembre, mientras que la temperatura alcanzó el valor más bajo en diciembre $\left(11,3^{\circ} \mathrm{C}\right)$ y el más alto $\left(22,0^{\circ} \mathrm{C}\right)$ en julio y agosto. El ITH que depende directamente de los dos parámetros anteriores fluctuó principalmente en función de la temperatura, alcanzando valores de 52,5 en diciembre y 69,0 en agosto (figura 1).

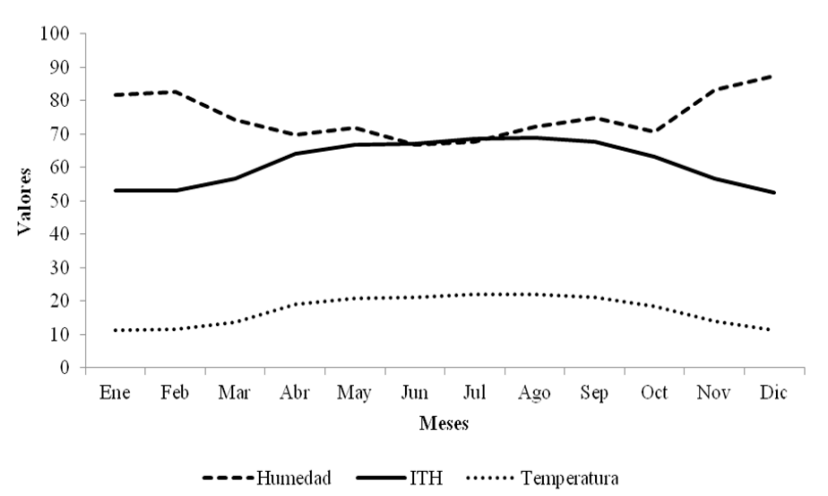

Figura 1. Variación de la humedad, temperatura e ITH a lo largo del año 2011 (Variation of humidity, temperature and ITH during the year 2011).

Se observó un patrón en los valores de ITH específica durante todo el día para cada estación del año. En cualquier momento el valor del ITH es siempre menor en el invierno, seguido del otoño y la primavera, presentando en verano los valores más altos para cualquier hora del día (figura 2).

Cuando se dividió el día en 6 períodos de 4-horas se constató que el valor más bajo de ITH se registró en el P2 entre las 4:00 y las 8:00 h $(47,4$ a 61,8) y el más 
elevado en el P4 (59,4 a 75,7) entre las 12:00 y las 16:00 $\mathrm{h}$, para todas las estaciones del año (figura 3).

En todas las explotaciones el aumento del ITH se refleja en la disminución de la producción de leche, con la excepción de la explotación 4 ( $p>0,05)$. En primavera, verano y otoño la producción de leche disminuye cada vez que hay un aumento de ITH, y en invierno ocurre exactamente lo contrario, es decir con el descenso del ITH se observó un ligero aumento en la producción diaria de leche/vaca (tabla III).

Todas las correlaciones fueron significativas $(\mathrm{p}<0,001)$ y de valor elevado (tabla IV), siendo las más relevantes entre la frecuencia respiratoria y el ITH $(0,83)$, entre la temperatura ambiente y la frecuencia respiratoria $(0,81)$, entre la temperatura rectal y el ITH $(0,79)$ y entre la temperatura ambiente y la temperatura rectal $(0,77)$.

Se observó una correlación positiva entre la frecuencia respiratoria y la temperatura rectal, que se cifró en 0,9 (figura 4). Se registró un efecto $(\mathrm{p}<0,005)$ del ITH en la producción de leche por día, cuando el valor del ITH era superior a 78 revelándose una menor producción del orden de 1,8 kg/vaca/día.

También se encontraron diferencias $(p<0,05)$ para otros parámetros estudiados (producción en ordeño, condición corporal, temperatura rectal y la frecuencia respiratoria) entre clases de ITH. La temperatura rectal y la frecuencia respiratoria sufrieron un incremento de $1,3^{\circ} \mathrm{C}$ y 37,3 movimientos/min respectivamente, cuando el ITH pasa de la clase inferior a 72 para la clase superior a 78 .

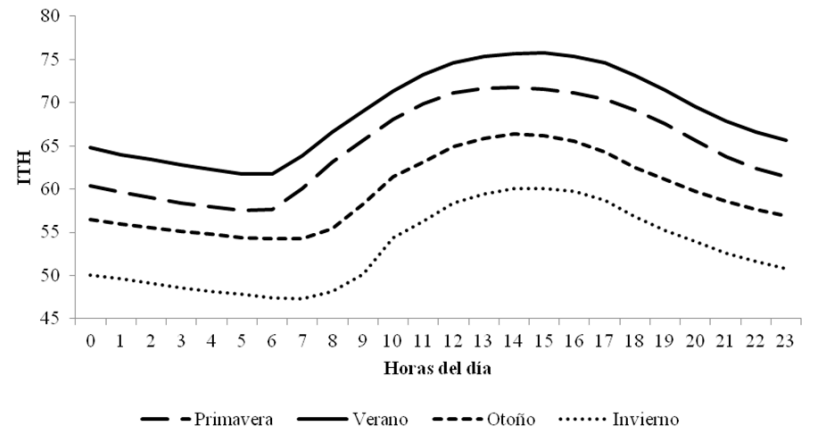

Figura 2. Variación del ITH a lo largo del día por estación del año (THI seasonal variation throughout the day).

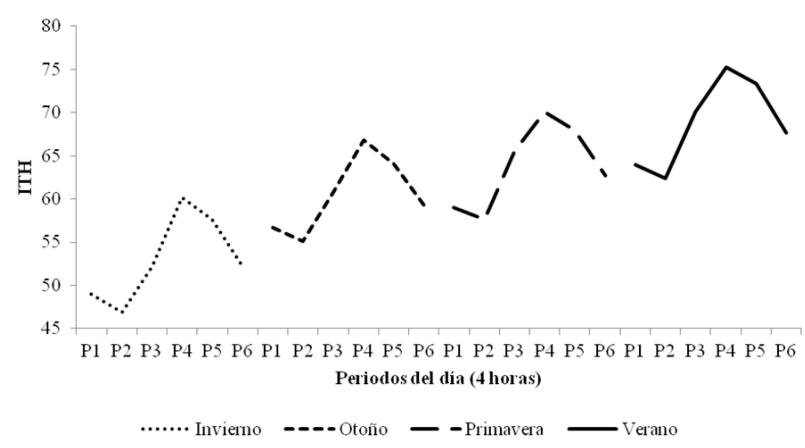

Figura 3. Variación de ITH a lo largo de las estaciones del año para 6 periodos del día (THI seasonal variation for six periods/day).

Tabla III. Coeficientes de regresión para las ecuaciones lineales simples entre la producción media diaria y el ITH en el período entre las 11 y las 17 horas (Regression coefficients for the linear equations between the average daily production and ITH in the time frame between 11 and 17 hours).

\begin{tabular}{|c|c|c|c|c|c|}
\hline Efectos & Indicadores & $\mathrm{N}$ & Coeficiente & Valor de $p$ & Producción (kg/dia) \\
\hline \multirow[t]{4}{*}{ Explotaciones } & 1 & 182 & $-0,15$ & 0,001 & 29,5 \\
\hline & 2 & 183 & $-0,08$ & 0,001 & 28,3 \\
\hline & 3 & 182 & $-0,09$ & 0,001 & 27,0 \\
\hline & 4 & 182 & $-0,03$ & $p>0,05$ & 26,4 \\
\hline \multirow[t]{4}{*}{ Estación del año } & Primavera & 184 & $-0,05$ & 0,01 & 29,0 \\
\hline & Verano & 183 & $-0,09$ & 0,05 & 26,4 \\
\hline & Otoño & 182 & $-0,09$ & 0,001 & 26,7 \\
\hline & Invierno & 180 & 0,14 & 0,001 & 29,1 \\
\hline \multirow[t]{12}{*}{ Meses de año } & 1 & 62 & 0,08 & $p>0,05$ & 29,0 \\
\hline & 2 & 56 & 0,12 & 0,05 & 29,4 \\
\hline & 3 & 62 & $-0,05$ & $p>0,05$ & 29,9 \\
\hline & 4 & 60 & 0,02 & $p>0,05$ & 29,6 \\
\hline & 5 & 62 & $-0,04$ & $p>0,05$ & 28,5 \\
\hline & 6 & 60 & 0,01 & $p>0,05$ & 28,2 \\
\hline & 7 & 62 & $-0,10$ & $p>0,05$ & 27,3 \\
\hline & 8 & 61 & $-0,18$ & 0,001 & 25,4 \\
\hline & 9 & 60 & $-0,19$ & 0,01 & 25,7 \\
\hline & 10 & 62 & $-0,11$ & 0,05 & 25,9 \\
\hline & 11 & 60 & 0,27 & 0,05 & 27,1 \\
\hline & 12 & 62 & 0,29 & 0,01 & 27,5 \\
\hline
\end{tabular}


Tabla IV. Correlaciones entre indicadores ambientales y fisiológicos (Correlations between environmental and physiological indicators).

\begin{tabular}{lcc}
\hline Indicadores ambientales & \multicolumn{2}{c}{ Indicadores fisiológicos } \\
\hline & $\begin{array}{c}\text { Temperatura } \\
\text { rectal }\end{array}$ & $\begin{array}{c}\text { Frecuencia } \\
\text { respiratoria }\end{array}$ \\
\hline Temperatura ambiente & $0,77^{\star * *}$ & $0,81^{* * *}$ \\
Humedad & $-0,63^{\star * *}$ & $-0,66^{\star * *}$ \\
ITH & $0,79^{\star * *}$ & $0,83^{\star * *}$ \\
\hline
\end{tabular}

ITH - índice de temperatura-humedad. Todos los valores de $r$ son significativamente $\left({ }^{* * *} p<0,001\right)$ diferentes de cero.

En la fase de lactación no se observaron diferencias ( $p>0,05)$, sin embargo, se constató una tendencia a una mayor producción diaria de leche, menor condición corporal, menor temperatura rectal y frecuencia respiratoria en el grupo de vacas cuya lactación era inferior a 60 días (tabla V).

\section{DISCUSIÓN}

El bienestar de las vacas lecheras y su desempeño productivo están relacionados con las condiciones ambientales del establo, especialmente en épocas de mayor calor. De hecho, hay evidencias de que la temperatura elevada del aire, especialmente cuando se asocia con una alta humedad relativa y intensa radiación solar son responsables de la disminución en la producción de leche, con un mayor impacto en las vacas de alta producción (Bouraoui et al., 2002; Kadzere et al., 2002;
West, 2003; Bohmanova et al., 2007; Bryant et al., 2007; Correa-Calderón et al., 2009; Rhoads et al., 2009; Wheelock et al., 2010; André et al., 2011). Nuestro estudio se ha basado en la utilidad de determinados indicadores fifio lógicos para medir la magnitud del efecto del estrés térmico. En general nuestros resultados están de acuerdo con los de los autores citados anteriormente para vacuno de leche. De hecho la gran especialización de estos animales en la producción de leche, traducida por su gran eficiencia en la utilización de los alimentos ingeridos, desencadena una alta producción de calor metabólico, haciéndolos más sensibles y más susceptibles al estrés por calor. Además, como resultado de su acción sobre la ingesta de alimentos, el estrés térmico influye en el metabolismo de la glándula mamaria y en la composición de la leche.

Los resultados obtenidos muestran un comportamiento típico según el patrón de la temperatura del aire de las estaciones estudiadas, donde los valores más críticos $\left(\geq 25^{\circ} \mathrm{C}\right)$ aparecen principalmente en verano y en primavera.

Durante el período de un año se contabilizaron 48 días con temperaturas superiores a $25^{\circ} \mathrm{C}$, valor límite a partir del cual las vacas lecheras sufren estrés térmico (McDowell et al., 1976; Fuquay, 1981; Roenfeldt, 1998; Kadzere et al., 2002; Overton et al., 2002).

Los valores de humedad relativa, fueron más altos en otoño e invierno (80,4\%). Cuando la humedad supera el $70 \%$, se produce una disminución del potencial de disipación del calor, lo que afecta a la producción de leche de los animales (Reece, 2009).

Tabla V. Influencia de la clase de ITH, número de lactación y fase de lactación en la producción, CC e indicadores fisiológicos $(\mathrm{n}=212)$ (Influence of class ITH, lactation number and stage of lactation on milk production, CC and physiological indicators $(n=212)$.

\begin{tabular}{|c|c|c|c|c|c|c|c|c|c|}
\hline Efectos & Indicadores & P. ord. & P. dia & P. 305 & Gr. & Prot. & $\mathrm{CC}$ & T. rectal & $\begin{array}{l}\text { Freq. } \\
\text { resp. }\end{array}$ \\
\hline \multirow[t]{2}{*}{ Clase ITH } & $\mathrm{ITH}<72$ & $16,2^{\mathrm{a}}$ & $34,8^{a}$ & $8851^{a}$ & $3,2^{a}$ & $3,1^{\mathrm{a}}$ & $2,7^{a}$ & $37,4^{a}$ & $43,4^{a}$ \\
\hline & $\mathrm{ITH}>78$ & $14,8^{b}$ & $33,0^{b}$ & $8864^{a}$ & $3,0^{\mathrm{a}}$ & $3,0^{a}$ & $2,6^{b}$ & $38,7^{b}$ & $80,7^{b}$ \\
\hline \multirow[t]{3}{*}{ Nº lactación } & 1 & $16,6^{a}$ & $36,0^{a}$ & $9018^{a b}$ & $3,2^{a}$ & $2,9^{a}$ & $2,7^{a}$ & $38,0^{a}$ & $60,2^{a}$ \\
\hline & 2 & $15,8^{a}$ & $34,8^{a}$ & $9127^{a}$ & $3,1^{\mathrm{a}}$ & $3,1^{b}$ & $2,7^{a}$ & $38,1^{a}$ & $63,2^{a}$ \\
\hline & $\geq 3$ & $14,1^{\mathrm{b}}$ & $30,9^{b}$ & $8427^{b}$ & $3,1^{\mathrm{a}}$ & $3,1^{\mathrm{ab}}$ & $2,5^{b}$ & $38,0^{a}$ & $62,7^{a}$ \\
\hline \multirow[t]{2}{*}{$\begin{array}{l}\text { Fase } \\
\text { lactación }\end{array}$} & $\leq 60$ días & $18,5^{\mathrm{a}}$ & $41,2^{\mathrm{a}}$ & $8469^{a}$ & $2,3^{a}$ & $2,6^{a}$ & $2,2^{a}$ & $37,2^{a}$ & $51,3^{a}$ \\
\hline & >60 días & $12,5^{\mathrm{a}}$ & $26,7^{\mathrm{a}}$ & $9246^{a}$ & $4,0^{\mathrm{a}}$ & $3,5^{\mathrm{a}}$ & $3,1^{\mathrm{a}}$ & $38,9^{a}$ & $72,7^{\mathrm{a}}$ \\
\hline \multirow[t]{5}{*}{ Probabilidad } & Cl. ITH & 0,028 & 0,041 & 0,214 & 0,072 & 0,964 & 0,01 & $<, 0001$ & $<, 0001$ \\
\hline & $\mathrm{N}^{\circ}$ lact. & 0,001 & 0,001 & 0,919 & 0,02 & 0,035 & $<, 0001$ & 0,485 & 0,386 \\
\hline & F. lact. & 0,441 & 0,344 & 0,412 & 0,333 & 0,829 & 0,196 & 0,115 & 0,463 \\
\hline & DL & 0,815 & 0,932 & 0,846 & 0,445 & 0,764 & 0,245 & 0,140 & 0,524 \\
\hline & DLxF.lact. & 0,582 & 0,418 & 0,845 & 0,299 & 0,357 & 0,249 & 0,138 & 0,592 \\
\hline \multirow[t]{3}{*}{ EPM } & Cl. ITH & 3,93 & 7,68 & 1,05 & 0,45 & 1801 & 0,36 & 0,51 & 14,62 \\
\hline & No lact. & 3,93 & 7,68 & 1,05 & 0,45 & 1803 & 0,36 & 0,51 & 14,63 \\
\hline & F. lact. & 4,05 & 7,92 & 1,08 & 0,46 & 1858 & 0,37 & 0,53 & 15,08 \\
\hline
\end{tabular}

P. ord.: producción/ordeño; P. día: producción/día; P. 305: producción a los 305 días; Gr.: grasa; Prot.: proteína; CC: condición corporal; T. rectal: temperatura rectal; Freq. resp.- frecuencia respiratoria; ITH: índice de temperatura-humedad; $\mathrm{Cl}$.: clase; $\mathrm{N}^{\circ}$ lact.: número de lactación; F. lact.: fase de lactación; DL: días en lactación; EPM: error de la media.

En los componentes analizados valores de letra distinta $(a \neq b)$ son significativamente diferentes $(p<0,05)$. 


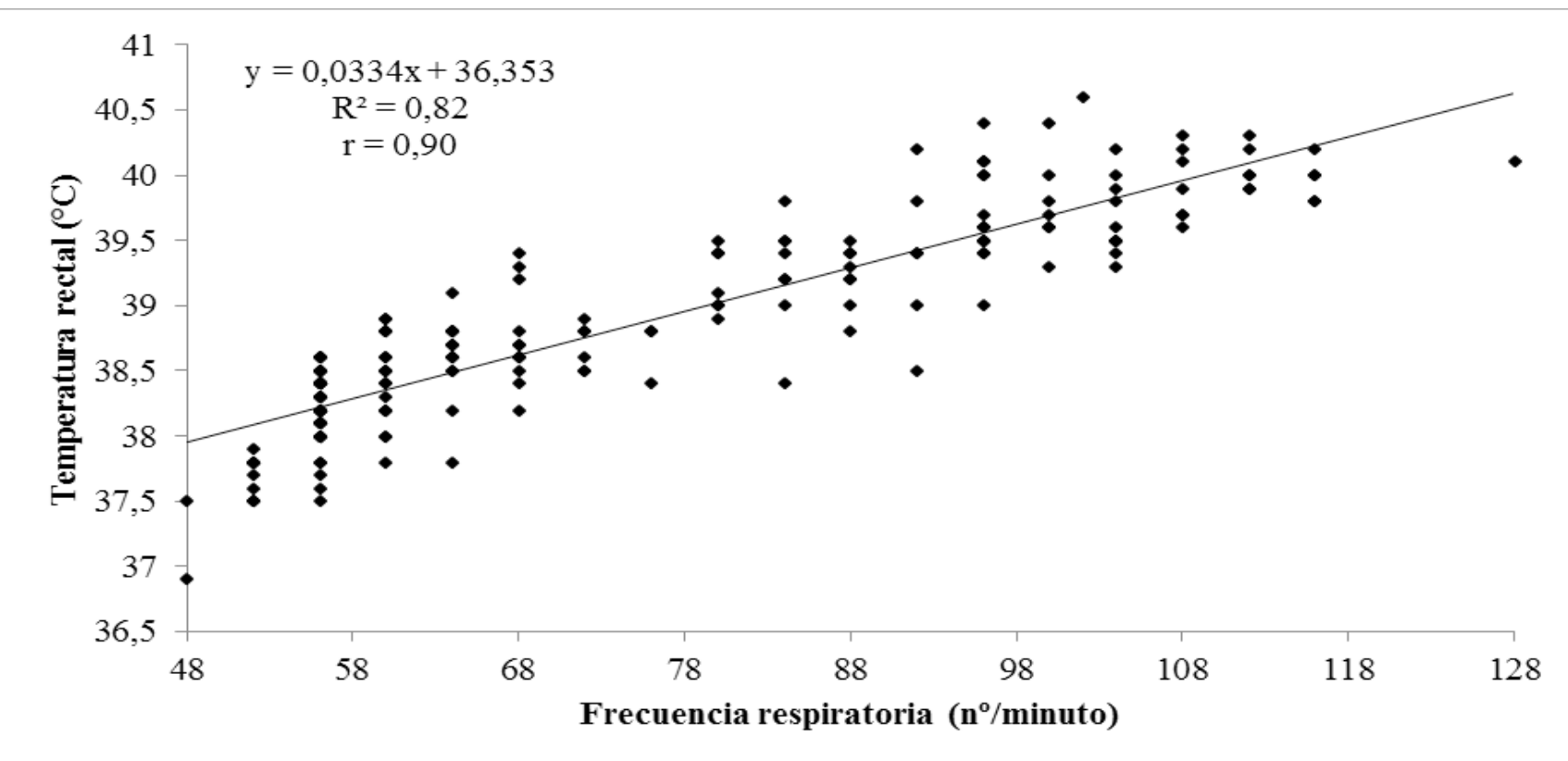

Figura 4. Regresión lineal entre la frecuencia respiratoria y la temperatura rectal (Linear regression between respiratory rate and rectal temperature).

Cuando el ITH supera el valor de 72, la producción de leche empieza a declinar (Johnson, 1985; DuPreez et al., 1990; Armstrong, 1994; Silva et al., 2002; Zimbelman et al., 2009), lo que se registró en 51 días del año, principalmente en el verano y primavera. Esta situación puede desencadenar una fuerte caída en la producción de leche, pues Ravagnolo et al. (2000) citan una reducción en la producción de $0,32 \mathrm{~kg}$ por unidad de incremento de ITH, por encima de 72. Según Zahner et al. (2004) con el aumento de ITH disminuye el tiempo de descanso de las vacas, que se puede asociar al aumento de frecuencia de cojeras (Cook et al., 2004).

El hecho de que el ITH tenga valores más elevados en la zona de comederos, comparativamente a la zona de los cubículos puede estar relacionado con la disposición de los equipos, porque el área del comedero suele estar en la parte lateral de los establos y de esta forma más expuestos a la radiación solar.

Aun así podría ser un factor positivo, teniendo en cuenta que los animales permanezcan más tiempo tumbados en los cubículos que alimentándose y por lo tanto están menos expuestos a condiciones climáticas adversas. Sin embargo, como no se encontraron diferencias en el ITH en la ubicación de las sondas en las estaciones de primavera y verano (más críticas para los animales) no hay interés en el análisis de los diferentes compartimentos del establo pero sí en su evaluación global.

En la investigación llevada a cabo durante los períodos del día, el valor más alto de ITH fue en el P4 (12:00 y 16:00 h), coincidiendo con el período de mayor intensidad de la radiación solar y de las temperaturas más altas, de acuerdo con el observado por CorreaCalderón et al. (2009).

Según estos resultados, es importante que en este período del día el ambiente del establo esté artificialmente controlado. West (1999), afirma que las alterna- tivas para mantener el rendimiento y bienestar de los animales en las estaciones calientes implica necesariamente una mejor adecuación de la administración de alimentos y una capacidad para facilitar a la disipación de calor animal en el medio ambiente.

Este enfriamiento se logra a través de sistemas de ventilación forzada, que pueden estar asociados con nebulización o aspersión de agua sobre los animales. Ya que la utilización conjunta de ventilación artificial y nebulización permite disminuir la temperatura del establo entre 8 a $12^{\circ} \mathrm{C}$ (Shearer et al., 1991).

La producción media diaria de leche por vaca, osciló entre 28 y $29 \mathrm{~kg}$ en primavera e invierno y 26 a $27 \mathrm{~kg}$ en otoño e invierno respectivamente, aunque durante el verano se esperaba encontrar una producción de leche inferior, debido a los valores más altos de ITH.

Los resultados corroboran los obtenidos por West (1999), cuando menciona que la producción de leche disminuye de 10 a $40 \%$ en el verano en relación con invierno. Según Barash et al., (1996) las vacas que parieron en el verano tuvieron menor producción de leche por lactación que las de invierno.

En otoño la menor producción de leche, puede explicarse por el hecho de que los meses de setiembre y octubre han revelado una temperatura y humedad superiores al patrón normal estándar de la zona de estudio para esos meses del año, lo que influyó en los valores de ITH.

Es posible que haya otros factores capaces de influir en la producción de leche, porque según Fuquay (1981) es difícil cuantificar los efectos ambientales directos en la producción de leche, siendo también afectada por la alimentación animal.

Con respecto a la respuesta de las vacas al estrés térmico, pueden ser citados indicadores hormonales (cortisol), de comportamiento o los indicadores fisio- 
lógicos, como la temperatura corporal y frecuencia respiratoria (Rushen et al., 2008; Correa-Calderón et al., 2009). Estos dos últimos son según Hemsworth et al (2000) indicadores muy interesantes para ser utilizados como una medida del confort animal. En las granjas bajo estudio había ligeras diferencias en los valores de temperatura rectal y la frecuencia respiratoria. Por otro lado, ambos parámetros sufrieron un incremento significativo en la transición de invierno a verano, fundamentalmente por la influencia en la subida del ITH.

Los resultados obtenidos son similares a los mencionados por Rhoads et al. (2009) y Wheelock et al. (2010), que citan aumentos de la temperatura rectal de 38,6 a $40,4^{\circ} \mathrm{C}$ y de 44 a 89 movimientos respiratorios por minuto en las vacas cuando entraron en estrés térmico. Tanto la frecuencia respiratoria como la temperatura rectal demostraron ser indicadores fiables del estrés térmico en los animales, ya que en los días de verano con temperaturas superiores a $34^{\circ} \mathrm{C}$, el $82 \%$ de las vacas superaban los 75 movimientos por minuto y el $73,0 \%$ una temperatura rectal por encima de los $39,1^{\circ} \mathrm{C}$. Estos resultados se corroboran con los de West (2003) y Correa-Calderón et al. (2009) en que la frecuencia respiratoria y temperatura rectal (más de 80 movimientos por minuto y $39,1^{\circ} \mathrm{C}$ respectivamente, muestran signos visibles de incomodidad térmica. También McDowell et al. (1976) aseguran que los aumentos de temperatura de $1^{\circ} \mathrm{C}$ son suficientes para reducir el comportamiento productivo. Los valores elevados en la frecuencia respiratoria y la temperatura rectal en verano también pueden explicarse por la alta correlación encontrada entre esos parámetros y el ITH, que fueron respectivamente de 0,83 y 0,79. Milam et al. (1985) recomiendan refrescar los animales con agua, lo que permite disminuir la frecuencia respiratoria de 90 a 81 movimientos por minuto.

Si por cuestiones operativas y de manejo fuese apropiado recoger sólo uno de los indicadores fisiológicos estudiados, se sugiere la observación de la frecuencia respiratoria, teniendo en cuenta que la correlación entre ambas variables es alta $(\mathrm{r}=0,90)$ y que es un parámetro que se monitoriza fácilmente en el ordeño.

Cuando el ITH supera el límite de 78 se observó una disminución de la producción del orden de 1,4 y 1,8 $\mathrm{kg}$ /ordeño por día respectivamente en cada vaca, que representa al menos una pérdida de producción diaria de unos $200 \mathrm{~kg}$ de leche en un lote de 100 vacas en lactación. Estos resultados son iguales que los obtenidos por Johnson (1985) al comprobar que la producción de leche disminuye cuando la temperatura supera los $38,9^{\circ} \mathrm{C}$ y por cada $0,55^{\circ} \mathrm{C}$ de aumento, la producción de leche y consumo de alimentos disminuyen 1,8 y 1,4 $\mathrm{kg}$ respectivamente. Datos similares fueron obtenidos por Reiczigel et al. (2009), Solymosi et al. (2010), Wheelock et al. (2010) y André et al. (2011) al afirmar que el estrés térmico produce una pérdida de producción del orden de 1,5 a 2,0 kg/vaca/día. También Bouraoui et al. (2002) informaron que el aumento de ITH de 68 a 78 condujo a una disminución en la producción de leche del $21 \%$.

A la vista de nuestros resultados coincidimos con Aguilar et al. (2009) al asegurar que las vacas se vuel- ven más sensibles al estrés térmico con un aumento del número de partos y que la creciente sensibilidad al estrés puede ser una de las razones para el deterioro de su vida productiva, especialmente bajo condiciones climáticas adversas.

La condición corporal factor que está estrechamente vinculado a la fase de lactación, reveló valores más bajos cuando el ITH fue mayor $(>78)$, lo que podría estar relacionado, como menciona West (2003), con una reducción en la capacidad de ingesta de alimentos implicando la pérdida de condición corporal. Scott et al. (1983) también refieren que elevadas temperaturas rectales conducen a la disminución del consumo de alimento.

\section{CONCLUSIÓN}

De acuerdo a lo revisado, la mejora genética para aumentar la producción de leche por vaca, aumenta la susceptibilidad al estrés térmico, por ellos debe mantenerse bajo control, principalmente en las estaciones cálidas del año, pues las pérdidas de producción de leche alcanzan los 1,8 kg/vaca/día.

El ITH más crítico para las vacas lecheras (>72) apareció en 51 días en el período anual y el período del día más susceptible que afecta al confort térmico de los animales es de 12:00 a 16:00, con registros medios de ITH de 68,0. Cuando el ITH supera el umbral del $<72$ a $>78$ hubo un incremento de temperatura de $1,3^{\circ} \mathrm{C}$ y de 37,3 movimientos por minuto por vaca.

Las altas correlaciones encontradas entre la frecuencia respiratoria, temperatura rectal con el ITH, sugieren estos indicadores fisiológicos excelentes como predictores de estrés.

\section{AGRADECIMIENTOS}

A los productores de leche que participaron en el proyecto. Este trabajo ha sido realizado con el apoyo de una beca mixta de doctorado FCT, en colaboración con el Instituto Politécnico de Viana do Castelo.

\section{BIBLIOGRAFÍA}

Aguilar, I.; Misztal, I. and Tsuruta, S. 2009. Genetic components of heat stress for dairy cattle with multiple lactations. J Dairy Sci, 92: 5702-5711.

André, G.; Engel, B.; Berentsen, P.B.M.; Vellinga, T.V. and Oude Lansink, A.G.J.M. 2011 . Quantifying the effect of heat stress on daily milk yield and monitoring dynamic changes using an adaptive dynamic model. J Dairy Sci, 94: 4502-4513.

Armstrong, D.V. 1994. Heat stress interaction with shade and cooling. J Dairy Sci, 77: 2044-2050.

Barash, H.; Silanikove, N. and Weller, J.I. 1996. Effect of season of birth on milk, fat and protein production of Israeli Holsteins. J Dairy Sci, 79: 1016-1020.

Bohmanova, J.; Misztal, I. and Cole, J.B. 2007. Temperature-humidity indices as indicators of milk production losses due to heat stress. J Dairy Sci, 90: 1947-1956.

Bouraoui, R.; Lahmar, M.; Majdoub, A.; Diemali, M. and Belyea, R. 2002. The relationship of temperature-humidity index with milk production of dairy cows in a Mediterranean climate. Anim Res, 51: 479-491. 
Bryant, J.R.; Lopez-Villalobos, N.; Pryce, J.E.; Holmes, C.W.; Johnson, D.L. and Garrick, D.J. 2007. Environmental sensitivity in New Zealand dairy cattle. J Dairy Sci, 90: 1538-1547.

Cook, N.B.; Nordlund, K.V. and Oetzel, G.R. 2004. Environmental influences on claw horn lesions associated with laminitis and subacuteruminal acidosis (SARA) in dairy cows. J Dairy Sci, 87(E Suppl.): E36-E46.

Correa-Calderón, A.; Santos, G.; Avendaño, L.; Rivera, F.; Alvarez, D.; Ardon, F.; Diaz, R. and Collier, R. 2009. Enfriamiento artificial y tasa de concepción de vaquillas holstein con estrés térmico. Arch Zootec, 58: 231-239.

Du Preez, J.H.; Giesecke, W.H. and Hattingh, P.J. 1990. Heat stress in dairy cattle and other livestock under Southern African conditions. I. Temperature-humidity indexmean values during the four main seasons, Onderstepoort. J Vet Res, 57: 77-86.

Fuquay, J.W. 1981. Heat stress as it affects animal production. J Anim Sci, 32: 164-174.

Hemsworth, P.H.; Barnett, J.L. and Beveridge, L. 1995. The welfare of extensively managed dairy cattle: a review. Appl Anim Behav Sci, 42: 161-182.

Hemsworth, P.H.; Coleman, G.J.; Barnett, J.L. and Borg, S. 2000. Relationships between human-animal interactions and productivity of commercial dairy cows. J Anim Sci, 78: 2821-2831.

Jacobsen, K.L. 1996. The well-being of dairy cows in hot and humid climates II. reducing stress. Compendium of Continuing Education in Practical Veterinary, vol. 18. Veterinary Learning Systems. Trenton. NJ. pp. S242-S254.

Johnson, H.D. 1980. Depressed chemical thermogenesis and hormonal functions in heat. In: Environmental physiology: aging, heat, and altitude. Elsevier /North Holland. New York. pp. 3-9.

Johnson, H.D. 1985. Physiological responses and productivity of cattle, In: Yousef M.K. (Ed.). Stress physiology in livestock. Basic principles. Vol. 1. 4-19. CRC Press. Boca Raton. Florida. pp. 4-19.

Kadzere, C.T.; Murphy, M.R.; Silanikove, N. and Maltz, E. 2002. Heat stress in lactating dairy cows: a review. Livest Prod Sci, 77: 59-91.

Kibler, H.H. 1964. Environmental physiology and shelter engineering. LXVII. Thermal effects of various temperature-humidity combinations on Holstein cattle as measured by eight physiological responses.

Martello, L.S.; Junior, H.S.; Silva, S.L.; Cesar, J.C. and Balieiro, J.C.C. 2009. Alternative body sites for heat stress measurement in milking cows under tropical conditions and their relationship to the thermal discomfort of the animals. Int J Biometeorology, 54: 647-652.

McDowell, R.E.; Hooven, N.W. and Camoens, J.K. 1976. Effects of climate on performance of Holsteins in first lactation. J Dairy Sci, 59: 965-973.

Milam, K.Z.; Coppock, C.E.; West, J.W.; Lanham, J.K.; Nave, D.H. and LaBore, J.M. 1985. Effects of drinking water temperature on production responses in lactating Holstein cows in summer. J Dairy Sci, 69: 1013-1019.

Overton, M.W.; Sischo, W.M.; Temple, G.D. and Moore, D.A. 2002. Using time-lapse video photography to assess dairy cattle lying behavior in a free-stall barn. J Dairy Sci, 85: 2407-2413.
Ramos, A.C. 2009. Cow confort, el bienestar de la vaca lechera. Servet editorial. Navarra, Espanha.

Ravagnolo, O.; Misztal, I. and Hoogenboom, G. 2000. Genetic component of heat stress in dairy cattle, development of heat index function. J Dairy Sci, 83: 2120-2125.

Reece, W.O. 2009. Body heat and temperature regulation. Functional Anatomy and physiological of domestic animals, 4th edition. Ed. Wilwy-Blakwell.

Reiczigel, J.; Solymosi, N.; Konyves, L.; Maróti-Agóts, A.; Kern, A. and Bartyik, J. 2009. Examination of heat stress caused milk production loss by the use of temperature-humidity indices. MagyAllatorv, 131: 137-144.

Rhoads, M.L.; Rhoads, R.P.; VanBaale, M.J.; Collier, R.J.; Sanders, S.R.; Weber, W.J.; Crooker, B.A. and Baumgard, L.H. 2009. Effects of heat stress and plane of nutrition on lactating Holstein cows: I. Production, metabolism, and aspects of circulating somatotropin. J Dairy Sci, 92: 1986-1997.

Roenfeldt, S. 1998. You can't afford to ignore heat stress. Dairy Manage, 35: 6-12

Rushen, J.; Passillé, A.M.; von Keyserlingk, M.A.G. and Weary, D.M. 2008. The welfare of cattle. Editor Clive Phillips, Published by Springer, Dordrecht, The Netherlands. $310 \mathrm{pp}$.

Scott, I.M.; Johnson, H.D. and Hahn, G.L. 1983. Effect of programmed diurnal temperature cycles on plasma thyroxine level, body temperature, and feed intake of Holstein dairy cows. Int J Biometeorol, 27: 47-62.

Shearer, J.K.; Bray, D.R.; Bucklin, R.A. 1991. The management of heat stress in dairy cattle. J Dairy Sci, 35: 330-345.

Silva, I.J.O.; Pandorth, H. and Acararo, J.R. 2002. Efeitos da climatização do curral de espera na produção de leite de vacas Holandesas. Rev Bras Zootecn, 31: 2036-2042.

Solymosi, N.; Torma, C.; Kern, A.; Maróti-Agóts, A.; Barcza, Z.; Konyves, L.; Berke, O. and Reiczigel, J. 2010. Changing climate in Hungary and trends in the annual number of heat stress days. Int J Biometeorol, 54: 423-431

West, J.W. 1999. Nutritional strategies for managing the heat - stressed dairy cow. J Anim Sci, 77 (Suppl. 2): 21-35.

West, J.W. 2003. Effects of heat-stress on production in dairy cattle. J Dairy Sci, 86: 2131-2144.

Wheelock, J.B.; Rhoads, R.P.; VanBaale, M.J.; Sanders, S.R. and Baumgard, L.H. 2010. Effects of heat stress on energetic metabolism in lactating Holstein cows. J Dairy Sci, 93: 644-655.

Zahoner, M.; Schrader, L.; Hauser, R.; Keck, M.; Langhans, W. and Wechsler, B. 2004. The influence of climatic conditions on physiological and behavioural parameter in dairy cows kept in pen stables. Anim Sci, 78: 139-147.

Zimbelman, R.B.; Rhoads, R.P.; Rhoads, M.L.; Duff, G.C.; Baumgard, L.H. and Collier, R.J. 2009. A re-evaluation of the impact of temperature humidity index (THI) and black globe humidity index (BGHI) on milk production in high producing dairy cows. Proceedings of the Southwest Nutrition Conference (ed. RJ Collier). University of Arizona. pp. 158-169. 